\section{Metformin in cancer}

Epidemiological research has established a link between the widely used insulin-lowering drug metformin and a decrease in cancer incidence. Previous studies have shown that metformin inhibits growth of cancer cell lines, and, now, a new study by Kevin Struhl and colleagues suggests that metformin inhibits the inflammatory response required for cancer cell transformation (Proc. Natl. Acad. Sci. USA, published online 31 December 2012; doi:10.1073/pnas.1221055110). In this study, the authors used an inducible model of cellular transformation in which signs of an inflammatory response are rapidly evident after induction, with NF-KB activation seen within 15 min. Treatment with metformin inhibited the NF-KB response, but only when added soon after induction, suggesting that metformin works at an early stage. Previous work had suggested that metformin selectively kills a cancer stem cell (CSC)-like population in mice, and the authors show that metformin selectively inhibits NF- $\mathrm{kB}$ and STAT3 activities in these CSC-like cells. Previous work also had suggested that, in combination with standard chemotherapeutic agents, metformin prolongs remission in mouse tumors. In the current study, the authors show that metformin blocks the inflammatory pathway in such tumors, and this inhibition was correlated with prolonged remission in mice. Future work should focus on defining the mechanisms by which metformin blocks the inflammatory response in cancer.

\section{Hotspots in autism}

A new study investigates the genome-wide patterns of de novo mutations (DNMs) in autism spectrum disorder (ASD) and suggests that the genomes of affected individuals may harbor mutational hotspots (Cell 151, 1431-1442, 2012). Jonathan Sebat and colleagues sequenced the whole genomes of ten pairs of monozygotic twins concordant for ASD and their parents. They found nonrandom positioning of DNMs, with an enrichment of closely spaced DNMs ( $<100 \mathrm{~kb}$ apart). The authors then investigated whether different sequence and chromatin features were associated with mutability, finding that DNase hypersensitivity, GC content, nucleosome occupancy, recombination rate, simple repeats and the trinucleotide sequence around the mutation site significantly influenced mutability. They identified broad genomic regions tens to hundreds of kilobases in length that showed highly elevated mutability (sevenfold higher than the rest of the genome), as well as 'warm spots' showing two- to threefold higher mutability. The authors found that many known autism-associated genes fell within the top $20 \%$ of highly mutable exons, and they suggest that hypermutability may be common in disease-related genes. Finally, they note that 34 of their observed DNMs affect protein-coding genes and noncoding RNAs. Together with previously reported DNMs in autism, two novel genes (KIRREL3 and GPR98) are identified that harbor more than one DNM.

$P F$

\section{Primordial germ cell reprogramming}

Primordial germ cells (PGCs) undergo epigenome reprogramming during their development from cells in the epiblast, migration and arrival at the embryonic gonad, but details of the dynamics of reprogramming during PGC development are not known. Now, Wolf Reik and colleagues report whole-genome bisulfite sequencing and RNA

Written by Orli Bahcall, Pamela Feliciano, Emily Niemitz \& Kyle Vogan sequencing of mouse epiblast cells at embryonic day (E) 6.5, as well as of mouse PGCs at E9.5, E10.5, E11.5, E13.5 and E16.5 (Mol. Cell $48,849-862,2012$ ). The authors found that there was extensive early global loss of DNA methylation between E6.5 and E9.5, but methylation was maintained at a select group of $\mathrm{CpG}$ islands in imprinted genes, $\mathrm{X}$-linked genes and genes involved in meiosis and gamete generation. These genes then lost methylation once PGCs had entered the genital ridges. Analysis of patterns of change in methylation showed evidence of both passive and active demethylation. The authors used transcriptome data to define 12 clusters of transcripts with consistent expression changes during PGC development. These clusters included gene networks involved in pluripotency and meiosis. They also detected a burst of LINE1 retroelement transcription in female E16.5 PGCs. This work provides insights into the regulation of pluripotency and reprogramming in PGCs.

EN

\section{TCF7L2 and liver function}

Variation in TCF7L2 confers susceptibility to type 2 diabetes, but the mechanisms underlying this association are poorly understood. Hans Clevers and colleagues now report a key role for $T c f 7 l 2$ in regulating the metabolic activity of hepatocytes (Cell 151, 1595-1607, 2012). The authors generated mice with a conditional knockout of $T c f 7 l 2$ in pancreatic beta cells and found that the mice exhibited normal glucose handling in response to various dietary states. In contrast, mice with constitutive knockout of $T c f 7 l 2$ died shortly after birth with severe hypoglycemia. Analyses of liver function in these mice identified pronounced defects in carbohydrate and lipid metabolism, accompanied by reduced expression of key metabolic enzymes. To further assess the role of $T c f 7 l 2$ in liver, the authors conditionally deleted $T c f 7 l 2$ in adult hepatocytes and found that this resulted in reduced glucose levels in response to fasting or a high-fat diet, due to defective hepatic gluconeogenesis. Conversely, they found that transient overexpression of $T c f 7 l 2$ in adult liver resulted in elevated glucose levels accompanied by induction of a subset of metabolic target genes. These findings identify the liver as a key site of TCF7L2 function that may underlie its role in disease risk.

KV

\section{MRSA evolution and spread}

Methicillin-resistant Staphylococcus aureus (MRSA) epidemics, a major cause of healthcare-associated infection, have been caused by a limited number of clones. The epidemic MRSA-15 (EMRSA-15) clone has been found primarily in hospital settings and has been associated with rapid spread over the past two decades. Matthew Holden and colleagues now report whole-genome sequencing and phylogenomic analysis of 193 ST22 isolates (EMRSA-15 clones are included within this MLST) collected from both hospital and community settings within 15 countries from 1990 to 2009, representing a wide geographic and temporal diversity. (Genome Res., published online 8 January 2013; doi:10.1101/gr.147710.112). Their analyses allow tracing of the origins and spread of this epidemic clone, which was first observed in the UK in the 1980 s and spread rapidly through the UK, Europe, Asia and Africa. They were able to explain observed resistance phenotypes on the basis of known resistance-conferring mutations for $>99 \%$ of those tested. They found a stepwise evolution of resistance and show a strong selective pressure exerted by specific commonly used antibiotics. They identified two nonsynonymous SNPs associated with the acquisition of fluoroquinolone resistance within an EMRSA-15 clade in the UK in the mid-1980s, which seems to be associated with an early epidemic spread. 